
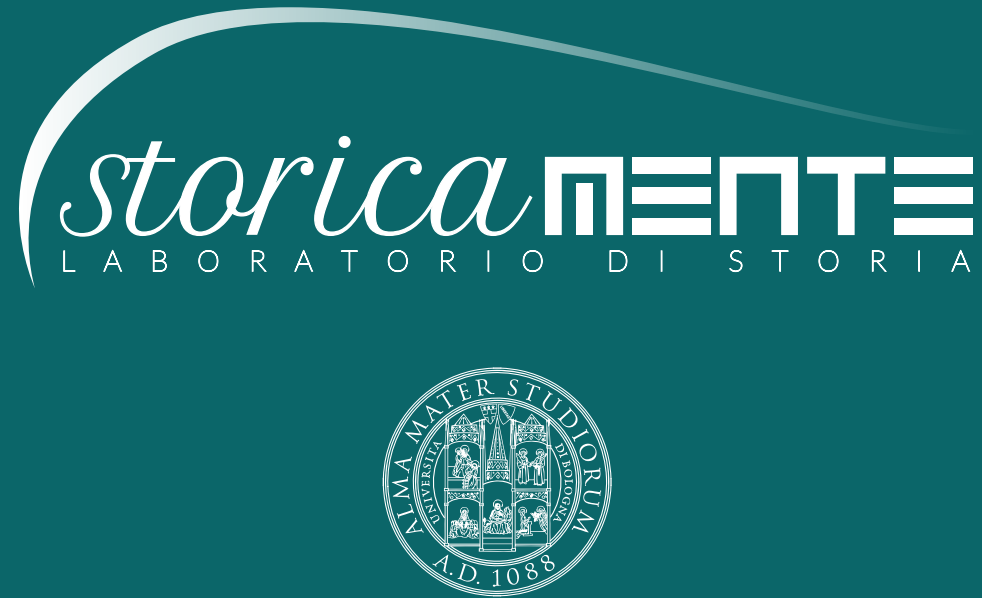

ALMA MATER STUDIORUM

Università di Bologna

Dipartimento di Storia Culture Civiltà

BIBLIO

TECA 


\section{STORICAMENTE.ORG}

\section{Laboratorio di Storia}

Angela De Benedictis

Pierre Girard, "“Comme des lumières jamais vues”. Matérialisme et radicalité politique dans les premières Lumières à Naples (1647-1744)"

Numero $13-2017$

ISSN: $1825-411 \mathrm{X}$

Art. 27

pp. 1-3

DOI: $10.12977 /$ stor679

Editore: BraDypUS

Data di pubblicazione: 06/11/2017

Sezione: Biblioteca 


\title{
Pierre Girard, "«Comme des lumières jamais vues». Matérialisme et radicalité politique dans les premières Lumières à Naples (1647-1744)"
}

\author{
ANGELA DE BENEDICTIS \\ Univ. Bologna, Dipartimento Storia Culture Civiltà
}

Pierre Girard, "“Comme des lumières jamais vues». Matérialisme et radicalité politique dans les premières Lumières à Naples (1647-1744)", Paris, Honoré Champion, 2016, 400 pp.

Una ricerca che ha lo scopo di avanzare una interpretazione generale della introduzione dei Lumi a Napoli alla data di morte di Giambattista Vico (1744) potrebbe apparire uno dei numerosi contributi su una problematica che certo continua a essere sempre storiograficamente attuale. Ciò che, invece, rende del tutto peculiare il libro del filosofo Pierre Girard è tanto il punto di partenza quanto il metodo seguito nella lettura congiunta di eventi e testi.

Il 1647, che nel luglio vede la "rivoluzione" di Masaniello e poi la continuazione del conflitto con la monarchia spagnola - attraverso la proclamazione della Real Repubblica Napoletana - fino all'esito dell'aprile 1648, costituisce il problema di partenza per l'analisi di Girard. Il perché sta nella chiave di lettura individuata e scelta per ripercorrere quel centinaio di anni: il materialismo e la radicalità politica segnalate fin dal titolo. Ciò significa, innanzitutto, accantonare in qualche modo l'idea che 
il pensiero meridionale italiano avesse qualità specificamente proprie. Comporta, invece, osservare - anche in base alle suggestioni dell'opera di Ernesto De Martino - la forma antimetafisica di quella cultura, il suo carattere concreto, politico, radicale, nonché il suo attaccamento all'esperienza e alla tradizione atomista e materialista, come conseguenza di una storia particolare che si rifrange in modo differenziato nell'insieme dell'esperienza umana, politica, sociale, scientifica o filosofica che sia. Metodologicamente diventa per questo necessario rinunciare al procedimento stesso della "ricostruzione" di idee, che implica una esposizione semplicemente lineare e orizzontale, e al relativo ricorso a una storia che giustifichi tutto tranne qualsiasi aspetto che possa mettere in pericolo la solidità dell'edificio, come il conflitto o il caso.

Le proprietà della storia che Girard osserva e analizza sono, al contrario, caratterizzate da quei meccanismi di rottura, di conflitto e di radicalità che contraddistinguono la varia "qualità dei tempi" nella Napoli di quel periodo. Nella storia delle idee il fare attenzione alla "qualità dei tempi" significa praticare nella lettura dei testi una "filologia politica" come quella che, ad esempio, è stata negli ultimi tempi utilizzata per Machiavelli, Guicciardini, Botero, Campanella (ma non solo) da altri studiosi francesi del pensiero politico italiano: Jean-Claude Zancarini, Jean-Louis Fournel, Romain Descendre.

La "rivoluzione" di Masaniello può allora essere il problema da cui prendere le mosse per via del mito costituito dalla figura del pescivendolo che diventa capo politico e militare di un movimento a favore del quale stanno i novatores in ambito specificamente scientifico-filosofico del tempo. Sono coloro che introducono i Lumi a Napoli nella seconda metà del Seicento, che si incontrano a partire dal 1649 in quella che sarà poi, dal 1663 al 1670, l' Accademia degli Investiganti: Tommaso Cornelio, Leonardo Di Capua, Francesco D’Andrea, Giuseppe Donzelli, Lucantonio Porzio, Giuseppe Valletta, Niccolò Sersale.

Sono medici, giuristi, filosofi le cui riflessioni e i cui testi sono suscitati sia da avvenimenti esteriori sia da altri testi ai quali reagiscono. L'a- 
tomismo e il pensiero materialista che li caratterizza rispondono a un doppio requisito della scienza e della politica. È l' “incertitudine” della medicina ufficiale di fronte alla peste del 1656 che impone loro la lotta contro i pregiudizi e le superstizioni scatenati dopo l'epidemia.

I medici ufficiali del Regno non sono in grado di diagnosticare la peste. Per paura, per ignoranza, per viltà davanti al potere dell'Inquisizione tardano ad ammettere l'esistenza della peste, persino a nominarla. Chi lo fa diventa simbolo della resistenza al potere dell'Inquisizione e al contempo di una libertas philosophandi concreta: in questo caso, nella lotta per una medicina efficace.

È nell'intreccio tra i campi scientifico, politico e religioso che consiste la particolarità napoletana, di cui appunto la rivoluzione di Masaniello rappresenta un momento decisivo. Di fronte alla peste la Chiesa usa politicamente la superstizione: molto rapidamente il clero fa notare che l'epidemia si è manifestata nel quartiere del Lavinaio, vicino alla piazza del Mercato, dove ha avuto inizio l'azione di Masaniello. Nel corso di sermoni improvvisati, i primi morti di peste sono presentati come vecchi rivoluzionari che avevano preso parte ai moti del 1647: la malattia non è altro che il giusto castigo di Dio. Soprattutto i Gesuiti occupano gli spazi pubblici annunciando la fine del mondo e il castigo divino. Davanti a una popolazione in preda al panico vengono presentati tutti una serie di eventi precedenti la peste come presagi della catastrofe: le epidemie di febbre del 1619-1621 e del 1625, l'eruzione del Vesuvio del 1631, i numerosi terremoti, la "empia" ribellione del 1647-1648, il passaggio di una cometa nel 1653. La risposta dei novatores è perciò scientifica, politica e religiosa, come Girard illustra nel suo denso libro, che va ben oltre l'esempio qui accennato della peste. $\mathrm{La}$ dimensione politica e scientifica della loro modernità, data la "qualità dei tempi”, consente loro peraltro di recuperare tradizioni antiche, come per esempio quelle atomistische, perché possono essere riattualizzate in funzione della loro capacità di rispondere ai bisogni e ai problemi del presente, in ogni campo del sapere e dell'esperienza.

In questo, il caso napoletano costituisce per l'a. un vero e proprio laboratorio. 